\title{
PREPARAÇÃO E CARACTERIZAÇÃO DE ÉSTERES E AMIDAS AROMÁTICAS SEGUINDO ALGUNS DOS PRINCÍPIOS DA QUÍMICA VERDE ${ }^{1}$
}

\author{
PREPARATION AND CHARACTERIZATION OF AROMATIC ESTERS AND \\ AMIDES FOLLOWING SOME OF THE PRINCIPLES OF GREEN CHEMISTRY
}

\author{
Daniel Victor Alves Rezende 2 , Bruno Alves Rezende 3 , \\ Marcos Vinícius Morais de Oliveira ${ }^{4}$ e Claudemir Batalini ${ }^{5}$
}

\section{RESUMO}

Ésteres e amidas constituem funções orgânicas clássicas em Química Orgânica. Tradicionalmente, em aulas práticas dessa disciplina, essas substâncias são preparadas utilizando ácidos inorgânicos fortes como catalisadores e sistema de aquecimento (refluxo). Este trabalho ressalta um caminho sintético alternativo e bem mais brando para a preparação de ésteres e amidas aromáticas, através do método de Schotten-Baumann, em meio aquoso básico e temperatura ambiente. Dois ésteres e três amidas aromáticas foram preparadas e caracterizadas por ponto de fusão, cromatografia em camada delgada (CCD) e espectroscopias no ultravioleta-visível (UV-VIS), no infravermelho (FTIR) e ressonância magnética nuclear de hidrogênio e de carbono (RMN'H e $\mathrm{RMN}^{13} \mathrm{C}$ ). Os rendimentos reacionais observados foram de satisfatórios a bons. Esta metodologia denotou um caminho sintético econômico, pouco agressivo à natureza, seguindo alguns princípios da Química Verde.

Palavras-chave: Funções orgânicas, Química sustentável, Schotten-Baumann, Síntese orgânica.

\section{ABSTRACT}

Esters and amides are classic organic functions in Organic Chemistry. Traditionally, in practical classes in this discipline, these substances are prepared using strong inorganic acids as catalysts and heating system (reflux). This work highlights an alternative and much milder synthetic path for the preparation of aromatic esters and amides, through the Schotten-Baumann method, in basic aqueous medium and at room temperature. Two esters and three aromatic amides were prepared and characterized by melting point, thin layer chromatography (TLC), ultraviolet-visible (UV-VIS), infrared (FTIR) and hydrogen and carbon nuclear magnetic resonance $\left({ }^{1} H N M R\right.$ and $\left.{ }^{13} C N M R\right)$ spectroscopies. Satisfactory to good yields were observed in the reactions. This methodology denoted a synthetic economic path, not very aggressive to nature, following some principles of Green Chemistry.

Keywords: Organic functions, Sustainable chemistry, Schotten-Baumann, Organic synthesis.

1 Trabalho de Iniciação Científica.

2 Mestre em Química Inorgânica - Universidade Federal de São Carlos - UFSCAR. E-mail: danielvic8@gmail.com

3 Mestrando em Química Orgânica - Universidade Estadual Paulista - UNESP/Araraquara-SP. E-mail: brunoalves_rezende@ hotmail.com

4 Graduado em Farmácia - Universidade Federal de Mato Grosso - UFMT/CUA. E-mail: marcos-ufmt@hotmail.com 5 Professor doutor (orientador) - Curso de Química Licenciatura - Universidade Federal de Mato Grosso - UFMT/CUA. E-mail: pirapotimao@msn.com 


\section{INTRODUÇÃO}

É muito frequente a inserção de práticas laboratoriais envolvendo síntese orgânica nas disciplinas de Química Orgânica nos cursos universitários do Brasil e do mundo. A preparação de ésteres e amidas, seja para a obtenção de aromas artificiais, fármacos e derivados são exemplos de sínteses bem comuns e que são exploradas no meio acadêmico, com destaque nos cursos de Química, Farmácia e Engenharia de Alimentos. Entretanto, geralmente essas sínteses envolvem um meio reacional agressivo, tanto para o meio ambiente, quanto para o experimentador, uma vez que são utilizados catalisadores constituídos de ácidos inorgânicos fortes (ácido sulfúrico $-\mathrm{H}_{2} \mathrm{SO}_{4}$ ou ácido fosfórico - $\mathrm{H}_{3} \mathrm{PO}_{4}$ ) e muitas vezes acompanhados de aquecimento (CAREY, 2011; MARCH; SMITH, 2013; OLIVEIRA et al., 2014). Uma rota alternativa para a preparação de ésteres e amidas aromáticas, em um meio reacional bem mais ameno, vem sendo utilizado com sucesso nas aulas experimentais de Química Orgânica II dos cursos de Química Licenciatura e Bacharelado em Farmácia da Universidade Federal de Mato Grosso - Campus Universitário do Araguaia (UFMT/CUA): constitui na clássica reação de Schotten-Baumann, conduzida em meio aquoso básico e temperatura ambiente (SCHOTTEN, 1884; BAUMANN, 1886; MARVEL; LAIZER, 1941; SOARES; SOUZA; PIRES, 1988; BALAJI; DALAL, 2018). Nessa reação, cloretos de ácido aromático podem combinar com fenóis e aminas aromáticas para a produção de ésteres e amidas aromáticas.

É cada vez mais visível a preocupação dos pesquisadores, seja dos meios acadêmicos ou das indústrias, com relação à preservação do meio ambiente, no que tange à elaboração de reações ou processos que gerem menor impacto ambiental (HAFEZ et al., 2013; BALAJI; DALAL, 2018; ZIMMERMAN et al., 2020). Toda ação nesse sentido diz-se que encontra-se alinhado com a chamada Química Verde, ou Green Chemistry, termo em inglês bastante disseminado na literatura científica. A expansão de trabalhos relatados na literatura sobre Química Verde é bastante significante, cabendo a Sousa-Aguiar e colaboradores (2014) um artigo específico sobre esse assunto.

Este trabalho segue, portanto, uma estratégia sintética mais racional e objetivou preparar, purificar e caracterizar dois ésteres aromáticos: benzoato de alfa-naftila (I) e benzoato de 8-quinolinila (II) e três amidas aromáticas: N-2-metilfenilbenzamida (III), N-8-quinolinilbenzamida (IV) e N,N'-(2,2'-dimetil-4,4'-difenil)-dibenzamida (V) (Figura 1).

\section{MATERIAL E MÉTODOS}

\section{Sínteses das substâncias}

Para as sínteses das cinco substâncias aromáticas utilizou-se a mesma metodologia (MARVEL; LAIZER, 1941; SOARES; SOUZA; PIRES, 1988). 
Em um erlenmeyer de $250 \mathrm{~mL}$, suspendeu-se 12,0 mmols dos fenóis alfa-naftol (para reação de síntese do éster (I)) e 8-hidroxiquinolina (para reação de síntese do éster (II)) ou 12,0 mmols das aminas 2-metilbenzeno (para reação de síntese da amida (III)), 8-aminoquinolina (para reação de síntese da amida (IV)) e orto-tolidina (para reação de síntese da amida (IV)) em $100 \mathrm{~mL}$ de solução aquosa de hidróxido de sódio $(\mathrm{NaOH})$ a $10 \%(\mathrm{~m} / \mathrm{v})$, à temperatura ambiente e sob agitação com barra magnética e após 20 minutos, 60,0 mmols de cloreto de benzoíla foram adicionados à solução. A reação foi mantida por mais 40 minutos sob as mesmas condições e os sólidos resultantes foram filtrados em funil de Büchner, lavados várias vezes com água destilada e os cristais foram secos em dessecador contendo sílica gel, sem a presença de vácuo.

Figura 1 - Esquema geral da preparação sintética dos ésteres (I) e (II) e das amidas (III) a (V).

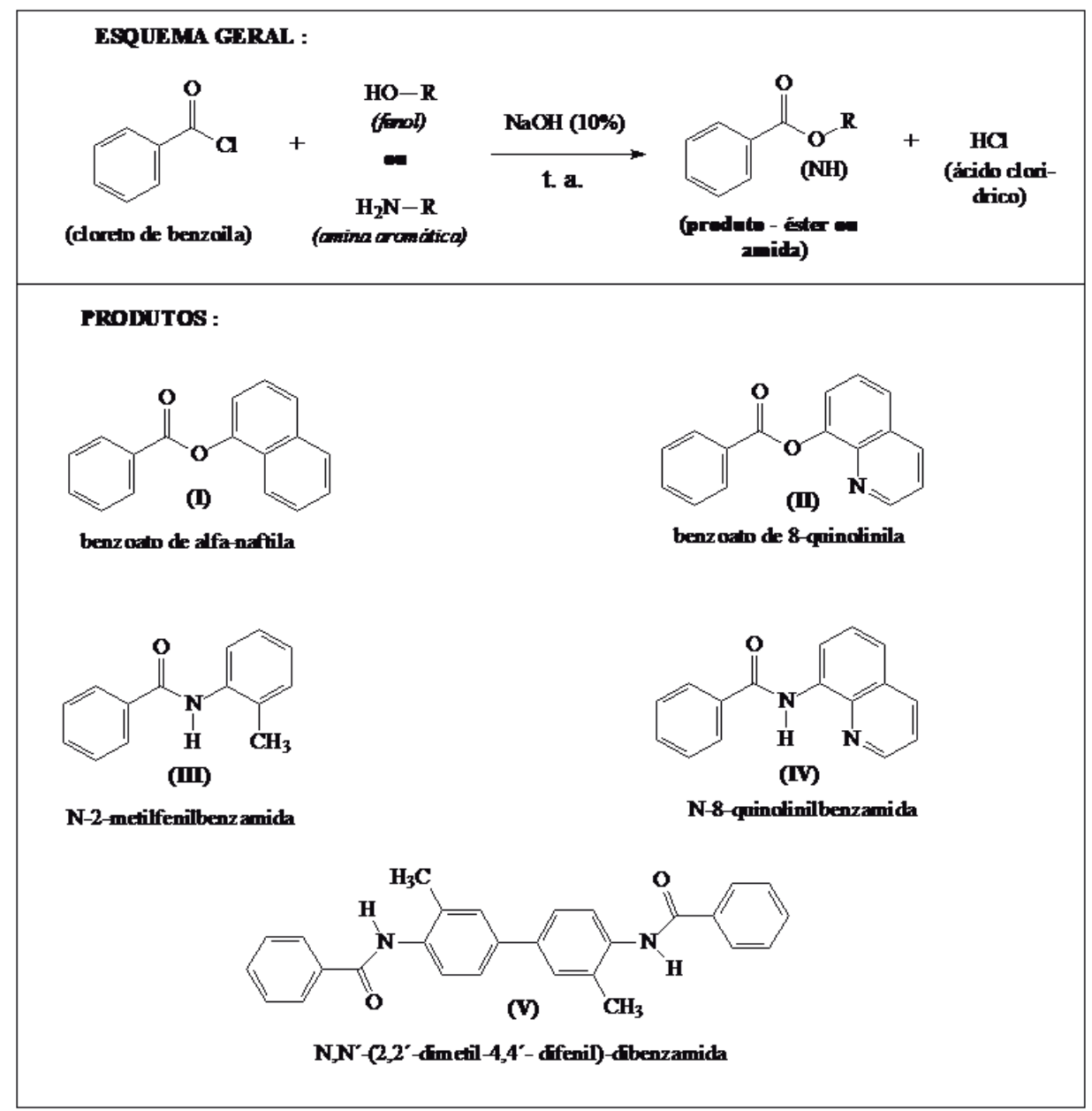

Fonte: Construção do autor.

\section{Purificações e Caracterizações dos produtos}

O éster (I) foi recristalizado em 1-butanol (Sigma), o éster (II) em água destilada e as amidas (III) a (V) foram recristalizadas em metanol (Aldrich). Os valores de pontos de fusão foram conseguidos manualmente em tubo de ensaio de $30 \mathrm{~cm}$ de altura e 3,0 cm de diâmetro, preenchido com 
glicerina (Sigma), acoplado a um termômetro. Para as análises de cromatografia em camada delgada (CCD) foram empregadas placas prontas de sílica gel G 60 (Mallinckrodt) como fase estacionária e clorofórmio (Merck) como fase móvel, observando as manchas que caracterizam as substâncias em câmara escura com lâmpada de ultravioleta-visível e também em cuba de vidro contendo cristais de iodo (Sigma) (COLLINS; BRAGA; BONATO, 1997). Espectros de ultravioleta-visível (UV-VIS) foram obtidos em espectrômetro Varian, modelo Cary 50 Scan, usando metanol (Aldrich) como solvente e espectros de infravermelho (FTIR) foram obtidos em espectrofotômetro com transformada de Fourier, modelo Perkin Elmer Spectrometer 100, resolução de $4 \mathrm{~cm}^{-1}$, na região compreendida entre 4000-600 cm-1 (PAVIA et al., 2010; SILVERSTEIN; WEBSTER; KIEMLE, 2005), usando cristais sem solubilização em solvente. Espectros de Ressonância Magnética Nuclear de Hidrogênio (RM$\mathrm{N}^{1} \mathrm{H}$ ) foram obtidos em aparelho Bruker Avance III HD $500 \mathrm{MHz}$ (solvente: clorofórmio deuterado - Aldrich) e os espectros de Ressonância Magnética Nuclear de Carbono 13 ( $\mathrm{RMN}^{13} \mathrm{C}$ ) foram obtidos em aparelho Bruker Avance III HD $126 \mathrm{MHz}$ (solvente: clorofórmio deuterado - Aldrich). Todos os reagentes e solventes usados neste trabalho encontravam-se na sua forma comercial P.A., sem nenhuma purificação adicional.

\section{RESULTADOS E DISCUSSÃO}

\section{Mecanismo de reação}

Nas Figuras 2 e 3 encontram-se os mecanismos de reação propostos para explicar as etapas previstas para as preparações das substâncias aromáticas (I) a (V) (SAVIN, 2014; SYKES, 2017). Num primeiro momento verifica-se que o mecanismo da reação de síntese de ésteres e amidas são muito semelhantes e envolvem o mesmo número de etapas. Na primeira etapa (Figura 2), o meio básico favorece a desprotonação do fenol, gerando um ânion fenóxi. Seguindo mecanismo $\mathrm{SN}_{2}$ (substituição nucleofílica bimolecular), o ânion fenóxi é fortemente básico para atacar a carbonila do cloreto de benzoíla, na segunda etapa, gerando um intermediário iônico com carbono $\mathrm{sp}^{3}$ tetraédrico, cuja estabilidade é intermediária. A terceira e última etapa é o do restabelecimento da carbonila, com retorno a um carbono de hibridização $\mathrm{sp}^{2}$ estabilizado, mediante a saída do cloro, grupo fortemente eletronegativo, formando assim o produto éster aromático.

Para as preparações das amidas aromáticas (III) a (V), o mecanismo de reação (Figura 3) segue os mesmos padrões relatados para a síntese dos ésteres aromáticos. 
Figura 2 - Mecanismo de reação geral para as sínteses dos ésteres (I) e (II).

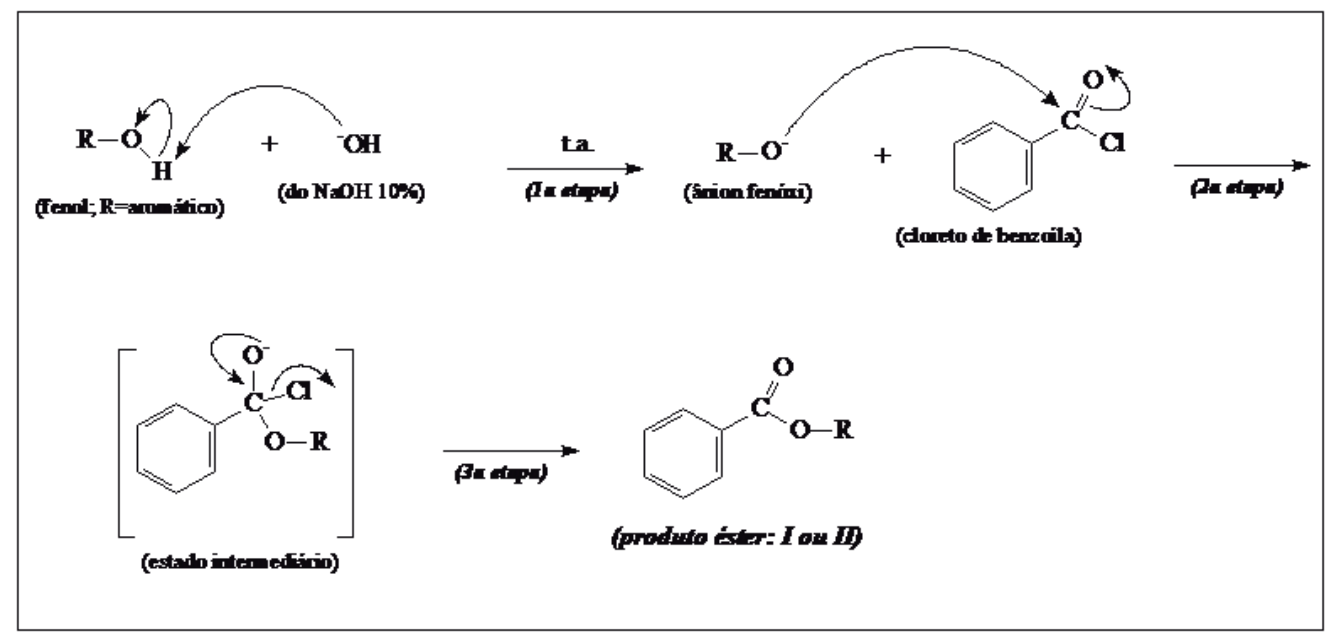

Fonte: Adaptado de Savin (2014); Sykes (2017).

Figura 3 - Mecanismo de reação geral para as sínteses das amidas (III) a (V).

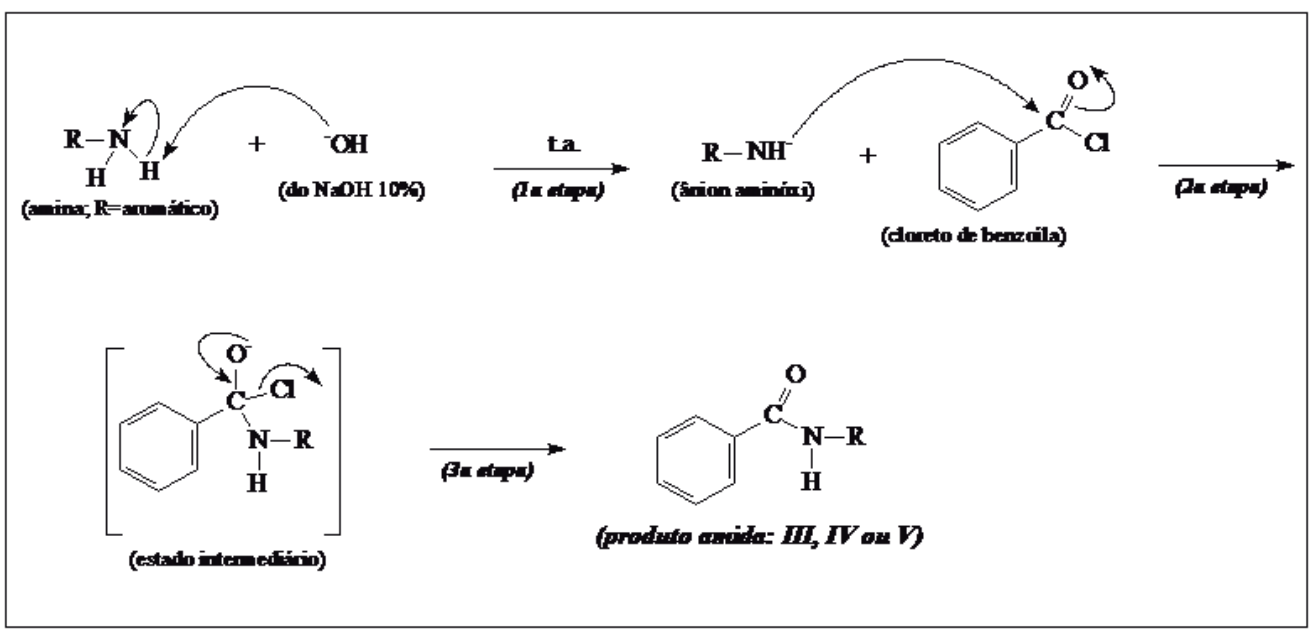

Fonte: Os autores (Referências: Savin (2014); Sykes (2017), com adaptações).

\section{Caracterizações dos produtos}

Na tabela 1 encontram-se os rendimentos reacionais após recristalização e alguns dados de caracterização dos ésteres e amidas sintetizadas.

Os rendimentos das reações revelaram-se de satisfatório a bom, com perdas esperadas após a recristalização. Apenas os rendimentos das preparações das três amidas situaram acima de 50\%. Quanto às cores dos cristais, o éster (I) apresentou cor amarelo areia, o éster (II) e a amida (III) apresentaram cristais brancos, a amida (IV) cor marrom e a amida (VIII) cor vermelha. Os valores dos pontos de fusão experimentais para as substâncias (I) a (IV) estão em concordância com os exibidos na literatura. Para a amida (V), não foi encontrado o valor experimental em busca na base de dados SciFinder, o que levanta a possibilidade de ineditismo estrutural. Nenhum dos valores de pontos de fusão foram coincidentes com os reagentes de partida (fenóis e aminas) empregados. Todas as subs- 
tâncias apresentaram uma única mancha visível em CCD, sendo o valor de fator de retenção diferentes dos reagentes de partida, atestando bom índice de pureza.

Os dados de UV-VIS das substâncias (Tabela 1) apresentam comprimento de onda máximo bem característico e esses dados não foram encontrados na literatura. Os dados de infravermelho, cujos principais estiramentos se encontram na Tabela 2, também não são descritos na literatura e os espectros são exibidos nas Figuras 4 a 8.

Tabela 1 - Dados de caracterização e rendimentos reacionais (\%) das substâncias sintetizadas.

\begin{tabular}{|c|c|c|c|c|}
\hline Produtos & $\begin{array}{l}\text { Pontos de } \\
\text { fusão }\left({ }^{\circ} \mathrm{C}\right)\end{array}$ & $\begin{array}{c}\text { Fator de } \\
\text { retenção (CCD) }\end{array}$ & $\begin{array}{l}\text { UV-VIS } \\
(\lambda \text { máx.) }\end{array}$ & Rendimentos (\%) \\
\hline \multirow{2}{*}{ benzoato de alfa-naftila (éster I) } & 55 (lit.) & \multirow{2}{*}{0,590} & \multirow{2}{*}{$262 \mathrm{~nm}$} & \multirow{2}{*}{30,6} \\
\hline & 56 (exp.) & & & \\
\hline \multirow{2}{*}{ benzoato de 8-quinolinila (éster II) } & 122 (lit.) & \multirow{2}{*}{0,140} & \multirow{2}{*}{$241 \mathrm{~nm}$} & \multirow{2}{*}{22,1} \\
\hline & 124 (exp.) & & & \\
\hline \multirow{2}{*}{ N-2-metilfenilbenzamida (amida III) } & 146 (lit.) & \multirow{2}{*}{0,070} & \multirow{2}{*}{$220 \mathrm{~nm}$} & \multirow{2}{*}{80,1} \\
\hline & 148 (exp.) & & & \\
\hline \multirow{2}{*}{ N-8-quinolinilbenzamida (amida IV) } & 84 (lit.) & \multirow{2}{*}{0,400} & \multirow{2}{*}{$240 \mathrm{~nm}$} & \multirow{2}{*}{67,7} \\
\hline & 85 (exp.) & & & \\
\hline \multirow{2}{*}{ N,N'-(2,2'-dimetildifenil)-dibenzamida (amida V) } & N/E (lit.) & \multirow{2}{*}{0,190} & \multirow{2}{*}{$222 \mathrm{~nm}$} & \multirow{2}{*}{81,2} \\
\hline & 318 (exp.) & & & \\
\hline
\end{tabular}

(lit.) = literatura; $($ exp. $)=$ experimental; $(\mathrm{N} / \mathrm{E})=$ não encontrado;

$(\lambda$ máx. $)=$ comprimento de onda máximo; $(\mathrm{nm})=$ nanômetros.

Tabela 2 - Principais estiramentos no infravermelho das substâncias sintetizadas (I) a (V).

\begin{tabular}{|c|c|}
\hline Produtos & Estiramentos no infravermelho $\left(\mathrm{cm}^{-1}\right)$ \\
\hline benzoato de alfa-naftila (éster I) & $\mathrm{C}=\mathrm{O}(1733), \mathrm{C}=\mathrm{C}(1400-1600), \mathrm{C}-\mathrm{O}(1221$ e 1089$)$ \\
\hline benzoato de 8-quinolinila (éster II) & $\mathrm{C}=\mathrm{O}(1730), \mathrm{C}=\mathrm{C}$ aromático $(1400-1600), \mathrm{C}-\mathrm{O}(1229$ e 1096$)$ \\
\hline N-2-metilfenilbenzamida (amida III) & N-H (3271), C-H (3044), C=O (1642), N-H (1513) e C-N (1318) \\
\hline N-8-quinolinilbenzamida (amida IV) & N-H (3352), C-H (3052), C=O (1671), N-H (1526) e C-N (1326) \\
\hline N,N'-(2,2'-dimetildifenil)-dibenzamida (amida $\mathbf{V})$ & N-H (3231), C-H (3060), C=O (1648), N-H (1486) e C-N (1309) \\
\hline
\end{tabular}

Figura 4 - Espectro de infravermelho do benzoato de alfa-naftila (éster I).

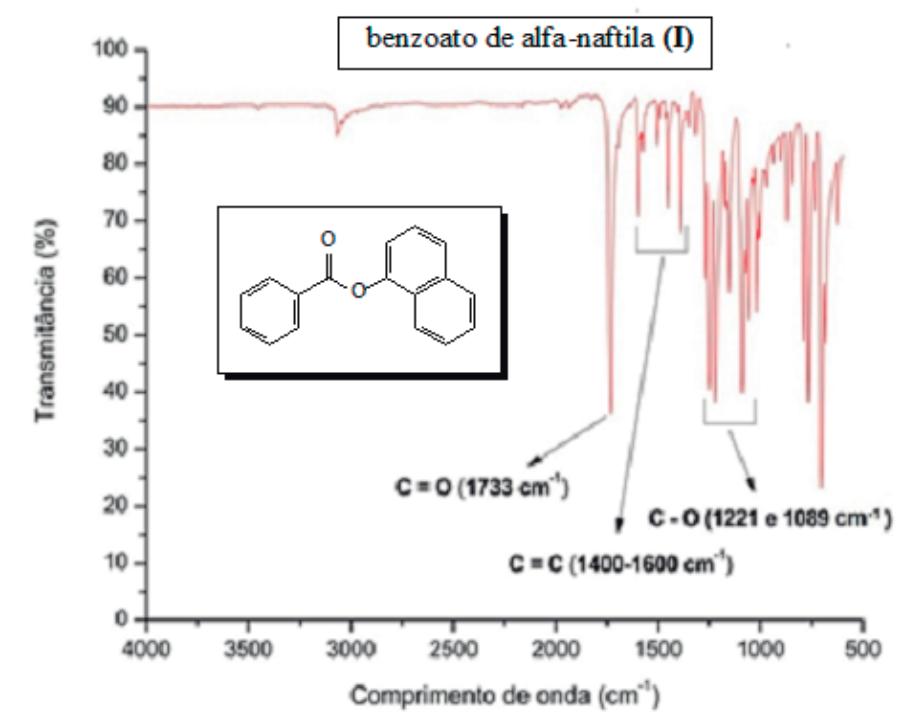

Fonte: Construção do autor. 
Figura 5 - Espectro de infravermelho do benzoato de 8-quinolinila (éster II).

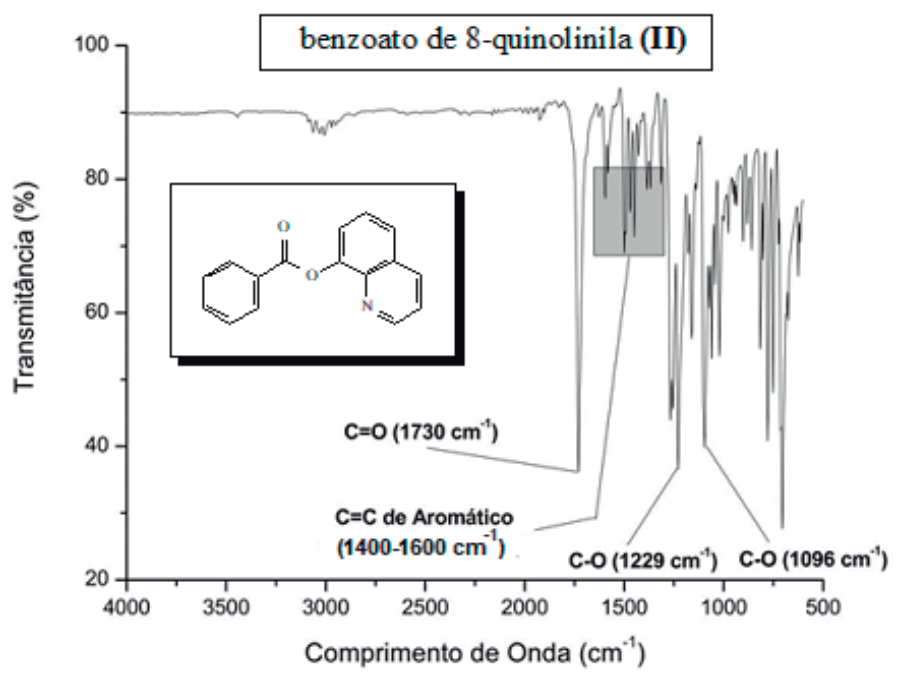

Fonte: Construção do autor.

Figura 6 - Espectro de infravermelho da N-2-metilfenilbenzamida (amida III).

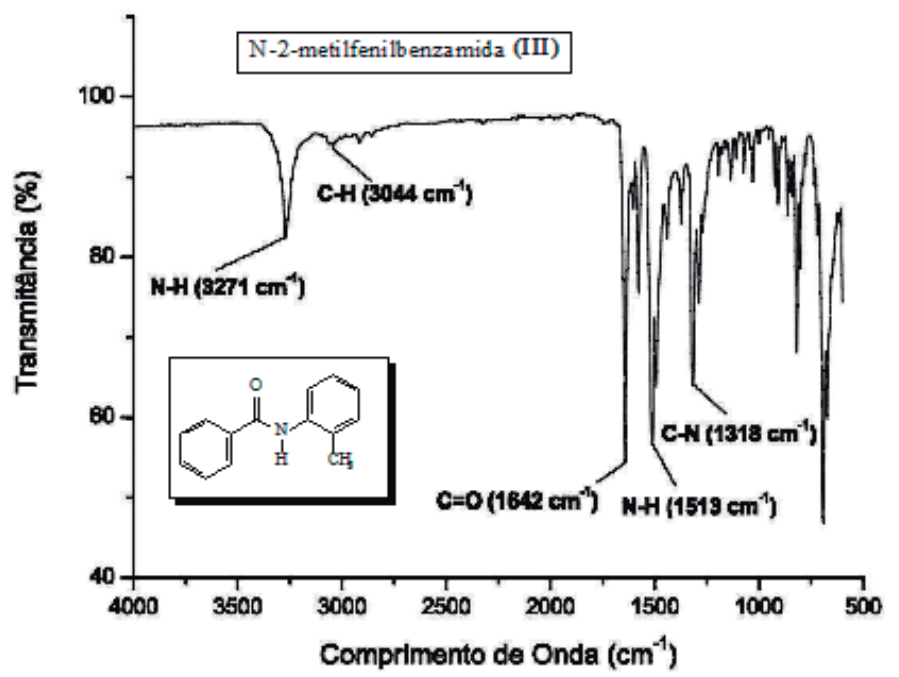

Fonte: Construção do autor.

Figura 7 - Espectro de infravermelho da N-8-quinolinilbenzamida (amida IV).

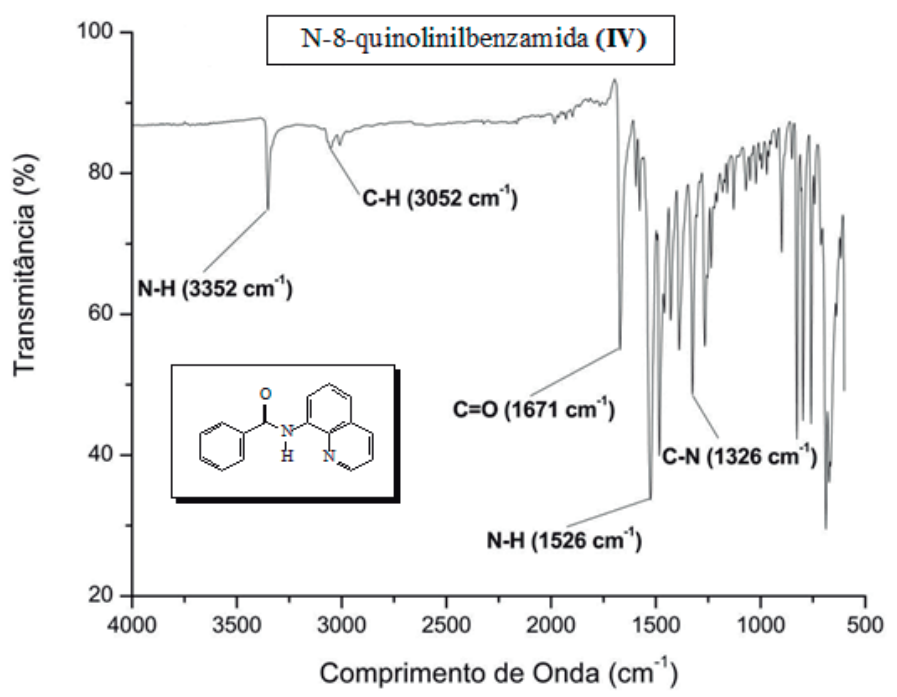

Fonte: Construção do autor. 
Figura 8 - Espectro de infravermelho da N,N'-(2,2'-dimetil-4,4'-difenil)-dibenzamida (amida IV).

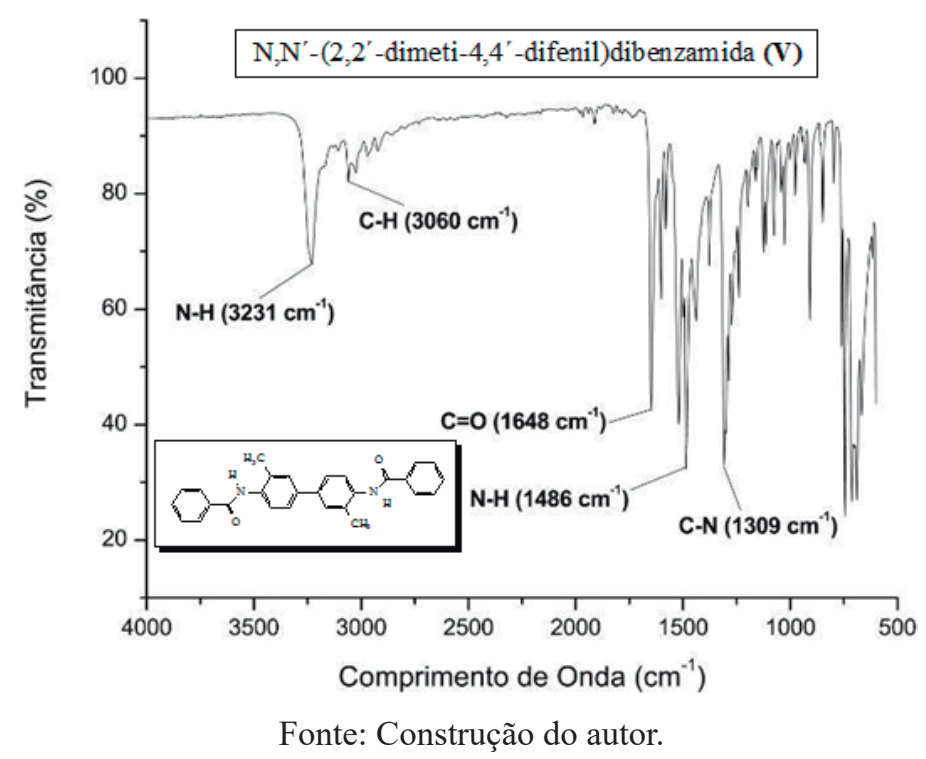

Análise dos espectros de infravermelho (FTIR) dos ésteres (I) e (II) (Figuras 4 e 5) permite descartar qualquer possibilidade de formação de ácido benzóico (como subproduto) ou um dos dois fenóis usados como reagentes de partida (alfa-naftol e 8-hidroxiquinolina), uma vez que não aparece em nenhum dos espectros o estiramento característico O-H (entre $3300-3100 \mathrm{~cm}^{-1}$ ); nesses dados, a presença do estiramento carbonila $\mathrm{C}=\mathrm{O}$, em $1730 \mathrm{~cm}^{-1}$, assegura a direção positiva da reação. Com relação aos espectros de FTIR das amidas (III) a (V), a presença do estiramento carbonila, um pouco abaixo de $1700 \mathrm{~cm}^{-1}$ também é um fator importante de que a reação se processou.

Os dados de $\mathrm{RMN}{ }^{1} \mathrm{H}$ e $\mathrm{RMN}^{13} \mathrm{C}$ obtidos para os produtos sintetizados (I) a (V) encontram-se na Tabela 3:

Tabela 3 - Sinais de $\mathrm{RMN}^{1} \mathrm{H}$ e $\mathrm{RMN}^{13} \mathrm{C}$ obtidos para as substâncias sintetizadas (I) a (V).

\begin{tabular}{|c|c|}
\hline Produtos & Sinais \\
\hline $\begin{array}{l}\text { benzoato de alfa-naftila } \\
\text { (éster I) }\end{array}$ & $\begin{array}{l}\text { FM: } \mathbf{C}_{17} \mathbf{H}_{12} \mathbf{O}_{2} \\
\mathbf{R M N}^{1} \mathbf{H}: 7,42(\mathrm{~d}, 1 \mathrm{H}) 7,51-7,61(\mathrm{~m}, 5 \mathrm{H}) 7,70-7,73(\mathrm{~m}, 1 \\
\mathrm{H}) 7,82(\mathrm{~d}, \mathrm{~J}=8,24 \mathrm{~Hz}, 1 \mathrm{H}) 7,93-7,95(\mathrm{~m}, 1 \mathrm{H}) 7,98-8,00 \\
(\mathrm{~m}, 1 \mathrm{H}) 8,38-8,40(\mathrm{~m}, 2 \mathrm{H}) . \\
\mathbf{R M N}^{13} \mathrm{C}: 77,41 ; 77,67 ; 118,62 ; 121,63 ; 125,85 ; 126,45 ; \\
126,84 ; 126,87 ; 127,36 ; 128,43 ; 129,10 ; 129,76 ; 130,68 ; \\
134,14 ; 135,08 ; 147,21 ; 165,55 .\end{array}$ \\
\hline $\begin{array}{l}\text { benzoato de 8-quinolinila } \\
\text { (éster II) }\end{array}$ & $\begin{array}{l}\mathbf{F M : ~} \mathbf{C}_{16} \mathbf{H}_{11} \mathbf{N O}_{2} \\
\mathbf{R M N}^{1} \mathbf{H}: 7,38(\mathrm{dd}, 1 \mathrm{H}) ; 7,51-7,61(\mathrm{~m}, 4 \mathrm{H}) ; 7,65(\mathrm{t}, 1 \mathrm{H}) \\
7,73(\mathrm{dd}, 1 \mathrm{H}) ; 8,15(\mathrm{dd}, 1 \mathrm{H}) ; 8,40, \mathrm{dd}, 2 \mathrm{H}) ; 8,89(\mathrm{dd}, 1 \mathrm{H}) \\
\mathbf{R M N}^{13} \mathbf{C}: 77,25 ; 77,50 ; 121,71 ; 121,79 ; 126,10 ; 126,31 ; \\
128,66 ; 129,59 ; 129,64 ; 130,62 ; 133,66 ; 136,11 ; 141,38 ; \\
147,76 ; 150,61 ; 165,57 .\end{array}$ \\
\hline
\end{tabular}


<smiles>Cc1ccccc1NC(=O)c1ccccc1</smiles>

N-2-metilfenilbenzamida

(amida III)<smiles>O=C(Nc1cccc2cccnc12)c1ccccc1</smiles>

N-8-quinolinilbenzamida

(amida IV)

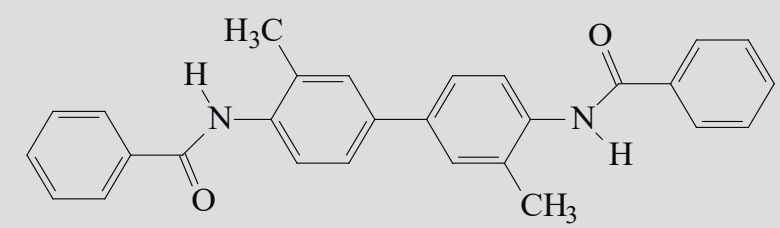

N,N'-(2,2'-dimetil-4,4' - difenil)-

dibenzamida

(amida V)
FM: $\mathrm{C}_{14} \mathrm{H}_{13} \mathrm{NO}$

RMN'H: 2,29 (s, 3H); 7,07-7,14 (m, 1H); 7,20 (d, 1H); 7,43 (t, 2H);7,49-7,56 (m, 1H); 7,85 (t, 4H); 10,57 (s, 1H). RMN ${ }^{13} \mathbf{C}: 17,86 ; 77,12 ; 77,37 ; 123,51 ; 125,51 ; 126,84$; 127,$14 ; 128,81 ; 129,78 ; 130,59 ; 131,84 ; 134,95 ; 135,80$; 165,83 .

FM: $\mathbf{C}_{16} \mathbf{H}_{12} \mathbf{N}_{2} \mathbf{O}$

RMN'1 H: 7,37 (dd, 1H); 7,46 (d, 1H); 7,52 (d, 4H); 8,07 (d, $3 \mathrm{H}) ; 8,77(\mathrm{~d}, 1 \mathrm{H}) ; 8,93(\mathrm{~d}, 1 \mathrm{H}) ; 10,69(\mathrm{~s}, 1 \mathrm{H})$

$\mathbf{R M N}^{13} \mathbf{C}: 77,23 ; 77,48 ; 116,50 ; 121,69 ; 121,73 ; 127,29$ 127,$38 ; 127,95 ; 128,81 ; 131,87 ; 134,54 ; 135,11 ; 136,34$; 138,$70 ; 148,27 ; 165,35$

FM: $\mathrm{C}_{28} \mathrm{H}_{24} \mathrm{~N}_{2} \mathrm{O}_{2}$

RMN'H: 2,31 (s, 6H); 7,07-7,14 (m, 2H); 7,17 (d, 2H); 7,43 (t, 4H); 7,47-7,55 (m, 2H); 7,86 (t, 6H); 10,40 (s, 2H). $\mathbf{R M N}{ }^{13} \mathbf{C}: 17,79 ; 76,90 ; 77,25 ; 123,12 ; 126,13 ; 126,65$; 127,$13 ; 128,44 ; 128,10 ; 130,78 ; 132,09 ; 135,15 ; 135,71$; 161,10 .

$\mathrm{RMN}^{1} \mathrm{H}$ (500 MHz, solvente: clorofórmio deuterado); $\mathrm{RMN}^{13} \mathrm{C}$ (126 MHz, solvente: clorofórmio deuterado); $\mathrm{s}=$ singleto; $\mathrm{d}=$ dupleto; $\mathrm{t}=$ tripleto; $\mathrm{q}=$ (quarteto) $\mathrm{m}=$ multipleto

Fonte: Os autores.

Os produtos e os processos da Química Verde se encaixam em três grandes categorias: emprego de matéria prima reciclável ou renovável, aumento da eficiência energética e eliminação de substâncias tóxicas (SEIDL et al., 2011). Dentro dessas três grandes áreas encontram-se doze princípios ou tópicos, que foram desenvolvidos quando se pretende implementar um processo com vistas à redução dos impactos ambientais (ANASTAS; WARNER, 1998, apud: LENARDÃO et al., 2003). As reações trabalhadas com os acadêmicos usando o método de Schotten-Baumann se alinham diretamente com a metade dos princípios da Química Verde (LENARDÃO et al., 2003), a saber: Princípio 1 - Prevenção, pois a metodologia empregada neste trabalho não gerou resíduos; Princípio 3 - Síntese de produtos menos perigosos, uma vez que as análises preliminares de potencial tóxico das substâncias preparadas indicam esse comportamento; Princípio 4 - Desenho de produtos seguros, que se alinha na verdade ao princípio 3; Princípio 5 - Solventes e auxiliares mais seguros, uma vez que o meio reacional utilizado foi aquoso e não houve a necessidade de empregar solventes de extração e sim apenas lavagem com água destilada; Princípio 6 - Busca pela eficiência de energia, com as reações sendo conduzidas em temperatura e pressão ambientes e Princípio 8 - Evita a formação de derivados, com todas as reações levando a apenas um único produto, conforme os dados exibidos neste artigo. Assim, 
o trabalho desenvolvido colabora no sentido de emprego de processo mais limpo, visando garantir os princípios de sistemas sustentáveis (ZIMMERMAN et al., 2020).

\section{CONCLUSÃo}

A substituição de métodos clássicos de preparação de ésteres e amidas aromáticas por uma rota alternativa, num ambiente mais limpo, simples e econômico, dentro da disciplina de Química Orgânica II, demonstrou eficácia e maior segurança para os alunos-experimentadores. Os rendimentos reacionais foram de satisfatórios a bons e a caracterização das substâncias apresentou boa concordância com a literatura e com as estruturas propostas. O grupo investiga a possibilidade da amida (V) apresentar estrutura inédita.

\section{AGRADECIMENTOS}

À UFMT - Campus Universitário do Araguaia e aos membros pesquisadores do LAPQUÍM Laboratório de Pesquisas em Química de Produtos Naturais - UFMT/CUA.

\section{REFERÊNCIAS}

ANASTAS, P. T.; WARNER, J. Green chemistry: theory and practice. Oxford: Oxford University Press, 1998.

BALAJI, B. S.; DALAL, N. An expedient and rapid green chemical synthesis of N-chloroacetanilides and amides using acid chloride under metal-free neutral conditions. Green Chemistry Letters and Reviews, v. 11, n. 4, p. 552-558, 2018.

BAUMANN, E. Ueber eine einfache methode der darstellung von benzoësäureäthern. Berichte der Deutschen Chemischen Gesellschaft, v. 19, n. 2, p. 3218-3222, 1886.

CAREY, F. A. Organic chemistry. 9. ed. New York (EUA): McGraw-Hill Higher Education, 2011.

COLLINS, C. H.; BRAGA, G. L.; BONATO, P. S. Introdução a métodos cromatográficos. 7. ed., Campinas (SP): Ed. da UNICAMP, 1997. 
HAFEZ, E. A. A.; AL-MOUSAWI, S. M.; MOUSTAFA, M. S.; SADEK, K. U.; ELNAGDI, M. H. Green methodologies in organic synthesis: recent developments in our laboratories. Green Chemistry Letters and Reviews, v. 6, n. 3, p. 189-210, 2013.

LENARDÃO, E. J.; FREITAG, R. A.; DABDOUB, M. J.; BATISTA, A. C. F.; SilveiRA, C. C. "Green chemistry" - os 12 princípios da química verde e sua inserção nas atividades de ensino e pesquisa. Química Nova, v. 26, n. 1, p. 123-129, 2003.

MARCH, J.; SMITH, M.B. Advanced organic chemistry: reactions, mechanisms, and structure. 7. ed. John Wiley \& Sons, 2013.

MARVEL, C. S.; LAIZER, W. A. Organic Syntheses. Coll. v. 1, p. 99; v. 9, p. 16, 1941.

OliveIRA, C. A.; SOUZA, A. C. J.; SANTOS, A. P. B.; SILVA, B. V.; LACHTER, E. R.; Pinto, A. C. Síntese de ésteres de aromas de frutas: um experimento para cursos de graduação dentro de um dos princípios da Química Verde. Revista Virtual de Química, v. 6, n. 1, p. 152-167, 2014.

PAVIA, D. L.; LAMPMAN, G. M.; KRIZ, G. S.; VYVYAN, J. R. Introdução à Espectroscopia. trad. 4. ed. norte-americana. São Paulo (SP): Cengage Learning, 2010.

SAVIN, K. Writing reaction mechanisms in organic chemistry. 3. ed. USA: Academic Press, 2014.

SEIDL, P. R.; BORSCHIVER, S.; FREIRE, E.; MOTA, C. J. A. Revista Brasileira de Engenharia Química,v. 27, p. 13, 2011.

SCHOTTEN, C. Ueber die oxydation des piperidins. Berichte der Deutschen Chemischen Gesellschaft, v. 17, n. 2 , p. $2544-2547,1884$.

SILVERSTEIN, R. M.; WEBSTER, F. X.; KIEMLE, D. J. Spectrometric identification of organic compounds. 7. ed. USA: John Wiley \& Sons, 2005.

SOARES, B. G.; SOUZA, N. A.; PIRES, D. X. Teoria e técnicas de preparação, purificação e identificação de compostos orgânicos. Rio de Janeiro (RJ): Guanabara, 1988. 
SOUSA-AGUIAR, E. F.; ALMEIDA, J. M. A. R.; ROMANO, P. N.; FERNANDES, R. P.; CARVALHO, Y. Química verde: a evolução de um conceito. Química Nova, v. 37, n. 7, p. 1257-1261, 2014.

SYKES, P. A guidebook to mechanism in organic chemistry. 6. ed. Pearson, 2017.

ZIMMERMAN, J. B.; ANASTAS, P. T.; ERYTHROPEL, H. C.; LEITNER, W. Designing for a green chemistry future. Science, v. 367, n. 6476, p. 397-400, 2020. 